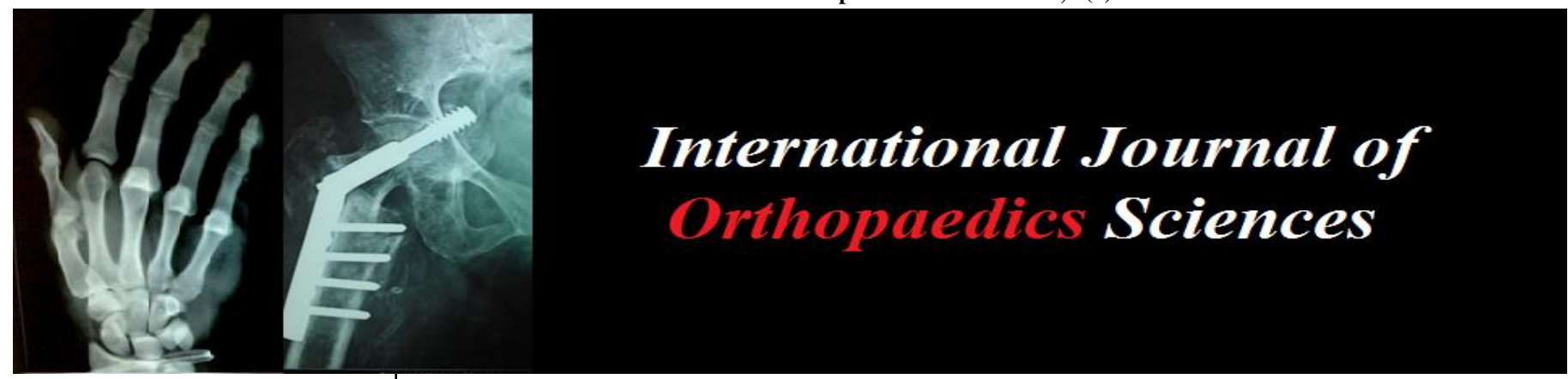

E-ISSN: 2395-1958

P-ISSN: 2706-6630

IJOS 2020; 6(4): 412-414

(C) 2020 IJOS

www.orthopaper.com

Received: 23-07-2020

Accepted: 27-08-2020

Dr. Ramavtar Saini

Professor \& Unit head,

Department of Orthopaedics, Geetanjali medical College and Hospital, Udaipur, Rajasthan India

Dr. Anshu Sharma

Assistant Professor, Department

of Orthopaedics, Geetanjali medical College and Hospital,

Udaipur, Rajasthan, India

Dr. Utkarsh Patel

$3^{\text {rd }}$ year Resident, Department of Orthopaedics, Geetanjali medica College and Hospital, Udaipur,

Rajasthan, India

Dr. Nihar Shah

Assistant Professor, Department of Orthopaedics, Geetanjali medical College and Hospital,

Udaipur, Rajasthan, India
Corresponding Author: Dr. Anshu Sharma

Assistant Professor, Department of Orthopaedics, Geetanjali medical College and Hospital, Udaipur, Rajasthan, India

\section{A case report on bilateral femoral neck fractures secondary to transient osteoporosis of pregnancy}

\author{
Dr. Ramavtar Saini, Dr. Anshu Sharma, Dr. Utkarsh Patel and Dr. Nihar \\ Shah
}

DOI: https://doi.org/10.22271/ortho.2020.v6.i4f.2367

\section{Abstract}

Pregnancy induced Transient osteoporosis of hip (TOH) is a rare, idiopathic self-limiting condition typically associated with the $3^{\text {rd }}$ trimester of pregnancy and early postpartum period. We describe a case of neglected atraumatic bilateral femoral neck fractures secondary to pregnancy induced Transient osteoporosis of hip. Fractures were diagnosed six months after delivery. Fixation was not feasible owing to poor bone quality and neck resorption, two stage cemented modular bipolar hemiarthroplasty were therefore performed for bilateral femoral neck fractures. Magnetic resonance imaging is the best noninvasive and safest investigative tool for pregnant women with hip pain and early detection can prevent complications and resorting to major surgeries.

Keywords: Pregnancy, transient osteoporosis of hip (TOH), fracture neck of femur, MRI, hemiarthroplasty

\section{Introduction}

Pregnancy induced transient osteoporosis is a rare, self-limiting, idiopathic skeletal disease. It present as loss of bone density and thinning of the cortex due to decrease mineralization of bone secondary to increased demand of pregnancy and decreased nutritional supply. ${ }^{1}$ It usually affects middle-aged women in the third trimester of pregnancy or primiparous women just after delivery ${ }^{[2]}$. The most common site involved is the hip (particularly on the left side) other sites include the vertebra, talus, knee, and acetabulum ${ }^{[3-5]}$. Among the hip cases 25 to $30 \%$ of the patients have bilateral hip involvement. Femoral neck fractures usually appeartwo months after the onset of symptoms, when the bone mass is at its lowest level ${ }^{[8]}$.

TOP usually presents with a sudden, quite severe onset of unilateral groin pain with no history of trauma. The patient may have an antalgic gait ormay be unable to walk ${ }^{[6-7]}$. Pain is elicited by hip rotation, although a full range of motion is common. Radiographs are avoided in pregnancy where possible, and are a poor investigation for demonstrating early osteopenia. DEXA scan is a good choice to detect poor mineralization and bone density. Magnetic Resonance Imaging (MRI) reveals low signal intensity of bone marrow on T1 weighted images, and high signal on T2 weighted images suggestive of bone marrow oedema ${ }^{[8]}$.

Fracture of femoral neck or acetabulam is rare in pregnant women with transient osteoporosis and bilateral fractures are evenrarer. We report a case ofpregnancy induced transient osteoporosis of hip leading to bilateral atraumatic femoral neck fractures that were diagnosed post-partum. Because of poor bone quality and neglected fractures with resorption of femoral neck, internal fixation was not feasible and two-stage cemented modular bipolar hemiarthroplasty were performed.

Case report: A 23-year-old women presented in orthopedic department with chief complaint of bilateral hip pain, difficulty in walking and weight bearing since four months without any history of trauma. After two months of delivery she developed pain in her left hip joint with difficulty in walking and was not investigated at that time. After few days she started to experience pain in her contralateral hip joint also. Patient was taking analgesics and continued walking with support. Patient presented to orthopedic OPD after fourmonths of starting pain when it was unbearable and walking was severely affected. 
The patient personal history doesn't reveal of any addiction, nor did she have been medicated for any anticonvulsant drugs or corticosteroids. She did not have any past and family history of thyroid, parathyroid, metabolic, bowel diseases or blood disorders (Sickle cell anaemia).

Plain radiograph of pelvis with both hip joints were taken after four months of initiation of symptoms and it revealed localized osteopenia, thinning of cortices with bilateral femoral neck fracture. (fig.1) Radiographs of wrist and spine (fig.2-3) were also suggestive of osteopenia and thinning of cortices. Serum calcium, phosphorus, alkaline phosphate, $24 \mathrm{hr}$ urinary calcium, liver, kidney, thyroid function testand other baseline investigation were within normal range.

Because of poor bone quality and neglected fractures with resorption of femoral neck, internal fixation was not feasible and two-stage bilateral cemented modular bipolar hemiarthroplasty were performed (fig.4). Postoperatively early ambulation and rehabilitation was started. Full functions were achieved gradually. Patient was advised for healthy diet with calcium and vitamin-D supplementation for three months.

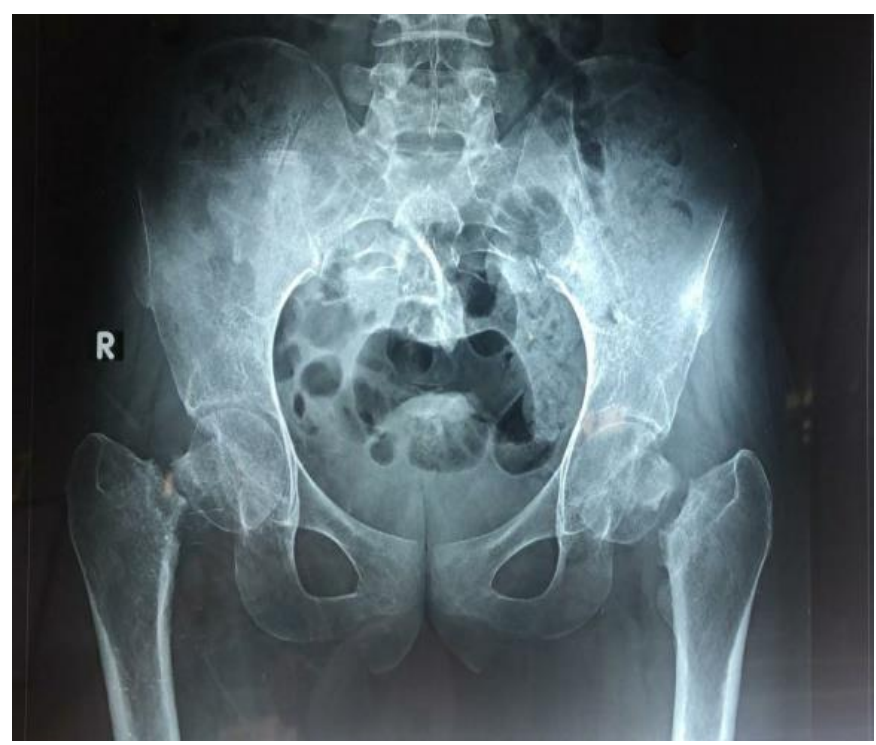

Fig 1.

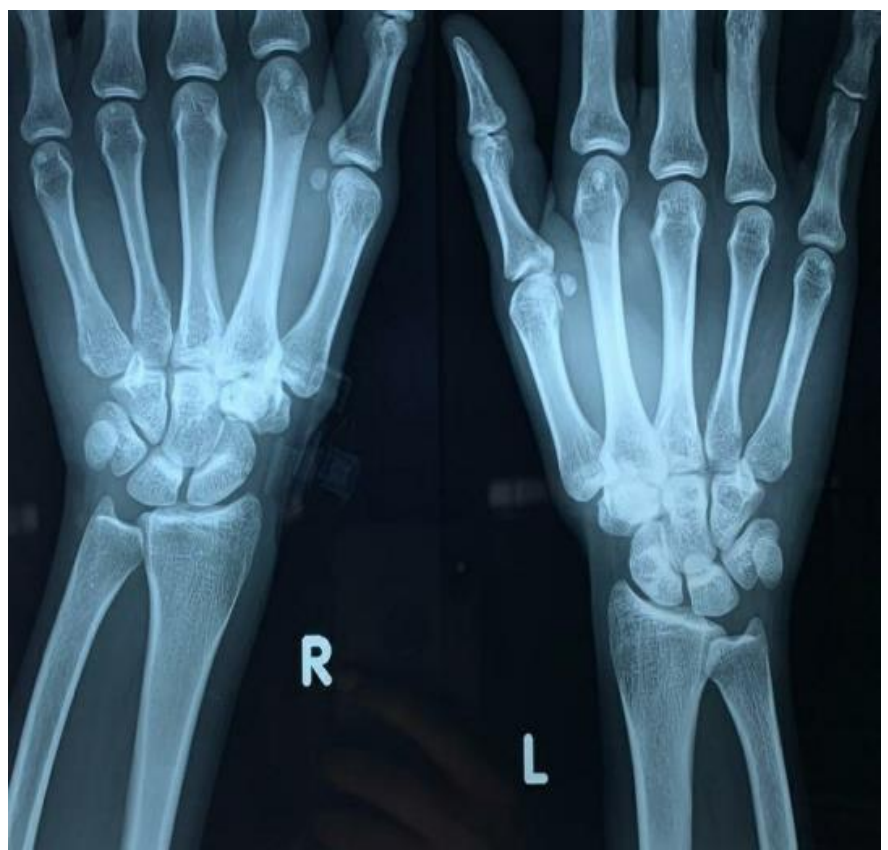

Fig 2.

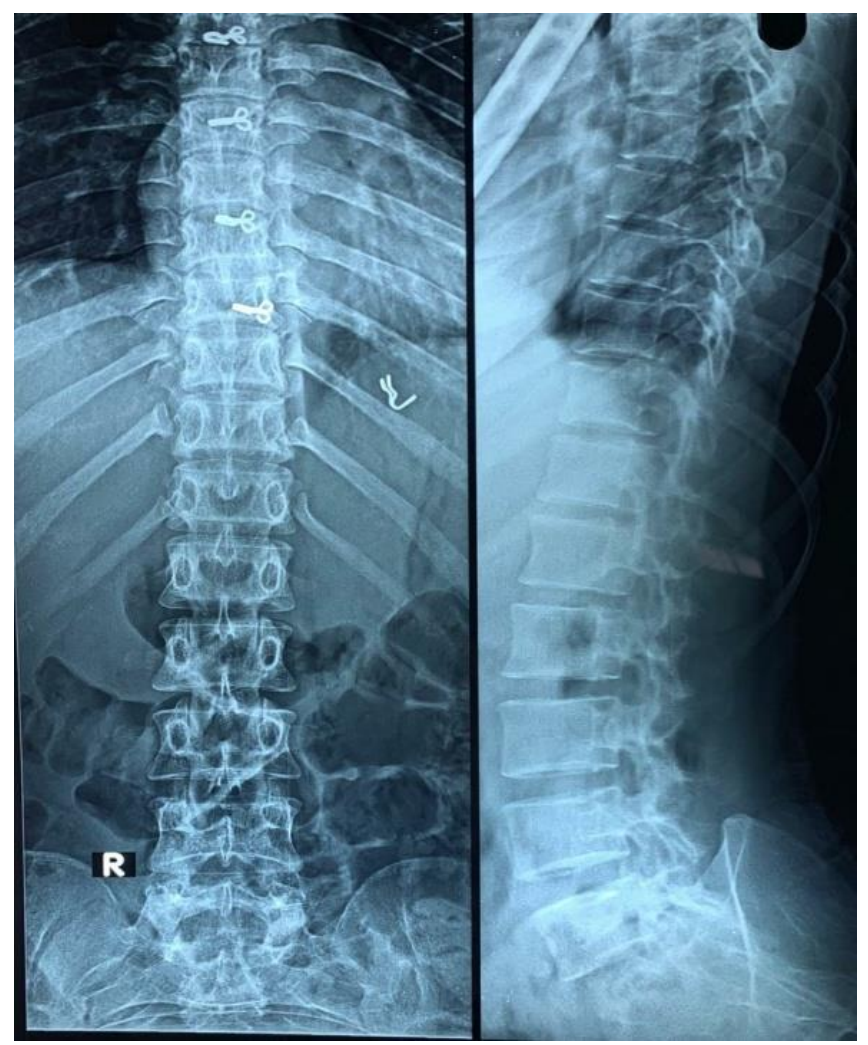

Fig 3.

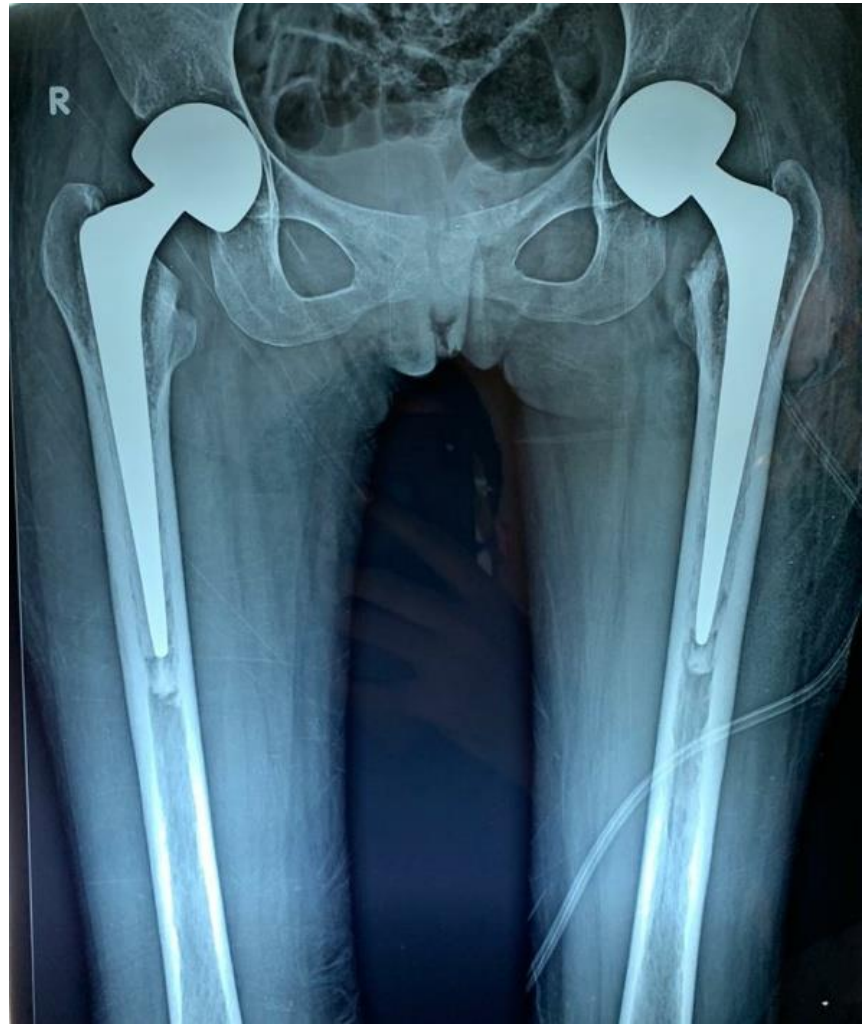

Fig 4.

\section{Discussion}

Transient osteoporosis of hip is a spontaneous skeletal disorder characterized by sudden onset of severe pain which resolves within 6-12 months. Transient osteoporosis of hip also known as bone marrow oedema syndrome, regional migratory osteoporosis, transient migratory osteoporosis, and hip algodystrophy. It occasionally affects lactating mothers and slender primiparous women in their third trimester of 
pregnancy. It usually affects a single or both hips (simultaneously or sequentially). Active and passive movement of hip are usually full and other laboratory investigation are usually within normal range. Recurrence in later pregnancy has also been reported ${ }^{[1,3]}$.

Its risk factors include poor nutrition, low calcium intake during pregnancy, and a family history of osteoporosis. The exact etiology is unknown; chemical, hormonal, mechanical, genetic, viral infections and neurovascular theories have been proposed ${ }^{[5]}$. During the first phase (first 2 months), hip pain and limp are noted; radiographs are normal and diffuse oedema is noted on MRI. In the second phase, symptoms increase and radiographs show osteoporosis with a normal joint space. Homologous focal lesions are specific MRI features. In the third phase (6 to 12 months), recovery and return to function can be expected, with MRI gradually returning to normal ${ }^{[6,7]}$.

Pregnant women frequently complain of hip or pelvic pain. The main differential diagnosis includes pubis symphysiolysis, inflammatory joint disorders, radiculopathy, soft tissue injury, reflex sympathetic dystrophy, osteonecrosis, septic arthritis, and rarely malignancy. A detailed history and meticulous clinical examination should be performed. In transient osteoporosis pain is of sudden onset which is aggravated by weight bearing and relieved by rest whereas in osteonecrosis pain is of insidious onset and not relieved by rest and gradually increases without spontaneous recovery ${ }^{[7]}$. In pregnant women who present with hip pain, radiographs are not much helpful in diagnosis of transient osteoporosis in early phases while MRI is the safest noninvasive tool. MRI changes in pregnancy induced transient osteoporosis of hipincludes diffuse edema, absence of focal defects, intact articular surface, absence of double line sign which is commonly associated with joint effusion. Decreased signal intensity of bone marrow in femur on $\mathrm{T} 1$ weighted images and increased signal intensity related to intensity ofnormal bone marrow $\mathrm{T} 2$ is seen typically ${ }^{[10]}$.

The management ofpregnancy induced transient osteoporosis of hip involves avoidance of weight bearing mainly as the involved bone is osteoporotic during the course and a risk of fracture femoral head or neck is always there. Analgesics should be given as and when required. Treatment approach for femoral neck fractures in young patients is usually head preserving (CRIF) but in our patient because of late presentation, poor bone quality and displaced fractured with resorption of femoral neck, internal fixation was not feasible and two-stage bilateral cemented modular bipolar hemiarthroplasty was performed.

\section{Conclusion}

This case report highlights the need for assessment of musculoskeletal complaints in pregnancy with detailed clinical examination. We suggest MRI should be considered for women presenting with significant hip pain in third trimester of pregnancy or in early post partum. Conservative management of suspected cases of pregnancy induced $\mathrm{TOH}$ should be advocated with a close follow up to avoid surgical intervention. Ideal treatment for young patient with femoral neck fractures is closed reduction and internal fixation but in pregnancy induced osteoporosis it depends on quality of bone, time of presentation and neck profile. Patient should encourage for regular calcium and vitamin-D supplementation during the course of pregnancy and lactation. patient for publication of this case report and accompanying images. A copy of the written consent is available for review by the Editor-in-Chief of this journal.

\section{References}

1. Cohen I, Melamed E, Lipkin A, Robinson D. Transient osteoporosis of pregnancy complicated by a pathologic subcapital hip fracture. J Trauma 2007;62:1281-3.

2. Chowdhury FU, Robinson P, Grainger AJ, Harris N. Transient regional osteoporosis: a rare cause of foot and ankle pain.Foot Ankle Surg 2006;12:79-83.

3. Debnath UK, Kishore R, Black RJ. Isolated acetabular osteoporosis in $\mathrm{TOH}$ in pregnancy: a case report. South Med J 2005;98:1146-8.

4. Ofluoglu O, Ofluoglu D. A case report: pregnancyinduced severe osteoporosis with eight vertebral fractures. RheumatolInt 2008;29:197-201.

5. Lloyd JM, Lewis M, Jones A. Transient osteoporosis of the knee in pregnancy. J Knee Surg 2006;19:121-3.

6. Ma FY, Falkenberg M. Case reports: transient osteoporosis of the hip: an atypical case. Clin OrthopRelat Res 2006;445:245-9.

7. Pai WC, Lin CY, Kao MJ, Lin FC. Transient osteoporosis of the hip during pregnancy: a case report. Tw J Phys Med Rehabil 2009;37:131-7.

8. Paraschou S, Makiev G, Anastasopoulos H, Flegas P, Karanikolas A. Transient osteoporosis of the hip: a report of 12 cases. EEXOT 2009;60:186-90.

9. Rishi V, Wahi P, Mahajan A. Transient osteoporosis of hip (migratory). JK Science 2008;10:194-6.

10. Vandeberg BC, Malghem JJ, Leucouvet FE, Janart J, Maldagne BE. Idiopathic Bone Marrow edema lesion of femoral head: Predictive value of MR imaging. Radiology 1999;212(2):527-35. 